\title{
Exploratory Analysis of COVID-19 Case Demographics in Gary, Indiana
}

\author{
Cameron Snapp ${ }^{1}$, Bill Trimoski ${ }^{1}$, Martin Brown ${ }^{2}$, Amy Han $^{3}$, and Tatiana Kostrominova ${ }^{4}$ \\ ${ }^{1}$ Indiana University School of Medicine; ${ }^{2}$ Gary Health Department and Gary Sanitary District; \\ ${ }^{3}$ Indiana University School of Medicine, Department of Psychiatry; ${ }^{4}$ Indiana University School of \\ Medicine, Department of Anatomy, Cell Biology and Physiology
}

\section{Background and Hypothesis:}

Health disparities are prevalent in Black populations, and COVID-19 is not an exception. COVID-19 is a pandemic that has been confirmed in 3.8 million Americans and has caused 133,283 deaths in the US (4/20/2020). Recent literature suggests that minoritized and impoverished populations are more severely impacted by COVID-19. Gary, Indiana has a large Black population (80\%), high number of residents living below the poverty line (34\%), and high unemployment rate (20\%). We hypothesized that Black individuals in Gary have a higher rate of positive cases, hospitalizations, and deaths than non-Black individuals. Also, we hypothesized that income (median household income measured by zip code) is negatively correlated with COVID-19 deaths.

\section{Experimental Design and Project Methods:}

In collaboration with the Gary Health Department, we analyzed demographic data on all positive cases in the city from 4/16/2020 through 6/19/2020. Case data was de-identified with 16 dimensions including age, race, sex, ethnicity, hospitalization, death, and zip code. Data was analyzed using Pearson's chi-square test and regression analysis.

\section{Results:}

Positive cases and hospitalizations are 2-fold and 3-fold more frequent in the Black population compared to the non-Black population in Gary $(p<0.0001, P<0.01$, age and populationadjusted), respectively. Median household income of a zip code is exponentially and negatively correlated with COVID-19 related deaths in that zip code $\left(R^{2}=0.7450, p=0.0123\right)$.

\section{Conclusion and Potential Impact:}

In Gary, there is a clear health disparity of both income and race, specifically in the context of COVID-19. Health officials can utilize this data to reallocate resources to highly populated, low income, and predominantly Black neighborhoods. In addition, future predictive analysis could be beneficial in developing a model to predict COVID-19 prevalence and severity. Such a model would help local health departments prepare for a second Covid-19 wave, providing for better outcomes for at risk populations through resource allocation. 